\title{
Effects of Continuous Cropping of Codonopsis tangshen on Rhizospheric Soil Bacterial Community as Determined by Pyrosequencing
}

\author{
Meide Zhang ${ }^{1}$, Yinsheng He ${ }^{1, *}$, Wuxian Zhou ${ }^{1}$, Lunqiang Ai ${ }^{1}$, Haihua Liu ${ }^{1}$, Liang Chen ${ }^{2, *}$ and Yan Xie ${ }^{2, *}$ (I) \\ 1 Institute of Chinese Herbal Medicine, Hubei Academy of Agricultural Sciences, Enshi 445000, China; \\ emailtoecho@163.com (M.Z.); zhou_wx222@163.com (W.Z.); 13402707171@139.com (L.A.); \\ lhh205521@sina.com (H.L.) \\ 2 CAS Key Laboratory of Plant Germplasm Enhancement and Specialty Agriculture, Wuhan Botanical Garden, \\ Chinese Academy of Sciences, Wuhan 430074, China \\ * Correspondence: hys-0001@163.com (Y.H.); chenliang888@wbgcas.cn (L.C.); xieyan@wbgcas.cn (Y.X.)
}

check for updates

Citation: Zhang, M.; He, Y.; Zhou, W.; Ai, L.; Liu, H.; Chen, L.; Xie, Y. Effects of Continuous Cropping of Codonopsis tangshen on Rhizospheric Soil Bacterial Community as Determined by Pyrosequencing. Diversity 2021, 13, 317. https:// doi.org/10.3390/d13070317

Academic Editors: Milko A. Jorquera and Jacquelinne Acuña

Received: 13 May 2021

Accepted: 9 July 2021

Published: 12 July 2021

Publisher's Note: MDPI stays neutral with regard to jurisdictional claims in published maps and institutional affiliations.

Copyright: (c) 2021 by the authors. Licensee MDPI, Basel, Switzerland. This article is an open access article distributed under the terms and conditions of the Creative Commons Attribution (CC BY) license (https:/ / creativecommons.org/licenses/by/ $4.0 /)$
Abstract: Codonopsis tangshen, a perennial herbaceous, has been shown to be affected by continuous cropping, with significant decline in both yield and quality. In this study, we studied the effect of continuous cropping on the abundance and composition of rhizospheric soil bacterial community. Results showed that continuous cropping causes a significant decline in both yield and quality. The nutrient content in continuous cropping soil was higher than that of soil in main cropping. Pyrosequencing analyses revealed Proteobacteria and Acidobacteria as the main phyla in two types of soils. Relative abundance of Acidobacteria, Nitrospirae, TM7, and AD3 phyla was observed to be high in continuous cropping soils, whereas Chloroflexi, Bacteroidetes, and Planctomycetes phyla were richer in main cropping soils. At the genus level, high relative abundance of Pseudomonas ( $\gamma$-Proteobacteria), Rhodanobacter, Candidatus Koribacter, and Candidatus were observed in continuous cropping soil. Different patterns of bacterial community structure were observed between different soils. Redundancy analysis indicated that organic matter content and available nitrogen content exhibited the strongest effect on bacterial community structure in the continuous cropping soil. Taken together, continuous cropping led to a significant decline in yield and quality, decrease in rhizospheric soil bacterial abundance, and alteration of rhizospheric soil microbial community structure, thereby resulting in poor growth of $C$. tangshen in the continuous cropping system.

Keywords: Codonopsis tangshen; continuous cropping; 454 pyrosequencing; bacterial community; bacterial structure; soil chemical properties

\section{Introduction}

Codonopsis tangshen (C. tangshen), an important medicinal plant, is highly valued in traditional Chinese medicine. It functions in strengthening organic immunity, dilating blood vessels, helping depressurization, improving microcirculation, and enhancing hematopoiesis [1]. This plant is applicable for treating anorexia, spleen deficiency, hyperirritability, palpitation, and lassitude ailments [2]. According to phytochemical analysis, C. tangshen contains lobetyolin, sterol, polysaccharides, phenylpropanoids, and saponin, besides other chemical compounds [3]. In China, C. tangshen could be a low-cost succedaneum for Panax ginseng, and dry roots of $C$. tangshen have been widely used as a tonic [4]. However, due to increasing disease pressure, continuous cropping of $C$. tangshen in the same plot of land leads to a significant decline in the yield and quality of the tubers also known as soil sickness, or continuous cropping obstacle problem [5]. Therefore, the factors leading to problems associated with continuous cropping of $C$. tangshen have become a research priority in China.

Many factors, such as the imbalance of soil nutrients, build-up of pests in the soil, disorder in physico-chemical soil properties, and shifts in microbial community, have been 
reported to cause the decline in crop yield and quality in continuous cropping regime [6]; autotoxicity of root exudates is considered as one of the main causes [7]. It is not only observed in consecutively cropped C. tangshen, but also in many other crops, and horticultural and medicinal plants. Terpenoids, phenolics, alkaloids, cyanogenicglycosides, and steroids are reported to be closely related to autotoxicity [8]. Previous studies have frequently used filter paper bioassays enriched with a single chemical to assess the allelopathic and ecological effects of root exudates in soil; however, they were considered inadequate and controversial as they excluded the effects of microbial communities and soil chemical properties [9]. In addition, many other studies showed that the autotoxicity of root exudates could shape the rhizosphere microbiology by attracting or deterring specific microbial species $[10,11]$. Therefore, the shifts in rhizosphere microbial community, under continuous cropping, have recently attracted considerable attention [12].

As is well-known, function of soil ecosystem is mainly governed by rhizosphere microbial and bulk microbial dynamics. Microbial composition and diversity affect humus formation and degradation, soil structure and biological interactions and geochemical cycles $[7,13]$. Root-related microbial communities influence many biochemical processes in the soil, thereby contributing to plant fitness. Autotoxicity of root exudates, and microbes affected by the rhizosphere secretions are harmful to plant health [14,15]. In addition, many studies reported that the continuous cropping problems of plants resulted from shifts in the soil microbial community induced by root exudates rather than direct allelopathic autotoxicity [16-18]. Increasing studies have shown that root exudates could select microorganisms in the rhizosphere, and that these plant-associated microorganisms could then influence plant growth and health [10]. Wu et al. found that consecutive monoculture of Pseudostellaria heterophylla can alter the fungal community in the soil, leading to an increase in pathogenic fungi [16]. Fusarium oxysporum, an important plant disease pathogen, increased with the increasing years of continuous cropping of Pseudostellaria heterophylla [5]. However, responses of the rhizosphere bacterial community (and their functional significance) to continuous cropping of $C$. tangshen have not yet been fully elucidated.

Next-generation sequencing technology generates a large number of DNA reads, as in 454 pyrosequencing, and has proven to be a suitable method for accurate microbial community analysis. Here, 454 pyrosequencing of the $16 \mathrm{~S}$ rRNA gene was employed to gain a deeper insight into the biodiversity of bacterial communities [19]. Therefore, this study was conducted to evaluate how the abundance and composition of soil bacterial community change with continuous cropping using 454 pyrosequencing. Moreover, we aimed to explore whether soil properties had any effect on the bacterial community structure.

\section{Materials and Methods}

\subsection{Field Experiment and Soil Sampling}

C. tangshen Oliv. cultivar 'Banqiao' (main varieties in the main production region) was used as the test material. The experiment was conducted at Enshi City, Hubei Province $\left(30^{\circ} 26^{\prime} \mathrm{N}, 109^{\circ} 23^{\prime} \mathrm{E}\right)$, which has a humid subtropical climate, annual mean temperature of $16.2^{\circ} \mathrm{C}$, and annual mean precipitation of $1600 \mathrm{~mm}$. The experiment included two regimes, main cropping (FLZ) and continuous cropping (LZ) of C. tangshen. For LZ regime, a field previously cultivated with maize for 7 years, and followed by 3 years of $C$. tangshen was selected, with the adjacent field previously cultivated with maize for 10 years being used as a control (FLZ). C. tangshen Oliv. was planted on 26 March 2014, and harvested by 19 September 2016. The rhizosphere soil samples of $C$. tangshen Oliv. at a depth of 0-30 cm were collected from both LZ and FLZ regimes on 4 May (named LZ1 and FLZ1), 14 July (named LZ2 and FLZ2), and 19 September (named LZ3 and FLZ3), 2016. Three plots $(1 \times 1 \mathrm{~m})$ are set up for each treatment, and $20 \mathrm{C}$. tangshen Oliv. plants are collected by the five-point sampling method in each plot, and the rhizosphere soils of 20 C. tangshen Oliv. plants are mixed into one replicate (Supplementary Figure S1). The rhizosphere soils of C. tangshen Oliv. were collected by shaking the roots and were separated into two parts. In detail, use a shovel to dig up the roots of $C$. tangshen Oliv. and remove large pieces of 
surface soil. Gently shake the root system three times, the fallen soil is non-rhizosphere soil, and the soil still sticking to the roots is rhizosphere soil. The rhizosphere soils were collected with a brush in a sterile self-sealing plastic bag and homogenized by being passed through a $2 \mathrm{~mm}$ sieve. One part was brought back to the laboratory within $10 \mathrm{~h}$ and stored at $-80^{\circ} \mathrm{C}$ until further processing for the experiments. The other part was air-dried at room temperature for two weeks for the determination of the physical and chemical properties of the soil. All treatments were carried out at the same site, to maintain consistency in soil and climatic conditions; the same fertilization and field management was carried out during the entire trial. Each treatment had three repeated plots, and the study area was completely random.

\subsection{Measurement of Yield and Lobetyolin Content}

Harvesting was performed in late September 2016. Plants from all plots were collected; fresh weight of each root was measured after each collection. The fresh roots were ovendried at $45^{\circ} \mathrm{C}$ for $96 \mathrm{~h}$, and then constant weight, before measuring the lobetyolin content. Lobetyolin content was determined by an HPLC method [20].

\subsection{Soil Chemical Characteristics}

Soil $\mathrm{pH}$ was determined by the potentiometric method and using a $\mathrm{pH}$ meter (innoLab 20P, Prima, UK). Total nitrogen was determined by the Kjeldahl digestion method and using AutoAnalyzer 3 (Seal, GER). Total phosphorus was determined after digestion in $\mathrm{HClO}_{4}$ and $\mathrm{H}_{2} \mathrm{SO}_{4}$ [21], and using AutoAnalyzer 3 (Seal, GER). Total potassium was determined after digestion in $\mathrm{NaOH}$ and using flame photometer (M410, Sherwood, UK). Exchangeable nitrogen was determined by using alkaline hydrolysis diffusion method. Available phosphorus was measured by Olsen method and with UV-1800 spectrophotometer (Shimadzu, Kyoto, Japan). Available potassium was measured by the ammonium acetate extraction method [22] and flame photometer (M410, Sherwood, UK). The organic matter content of soil was estimated by the potassium dichromate volumetric method.

\subsection{DNA Extraction, PCR and 454 Pyrosequencing}

Total DNA was extracted from soil samples using TruSeq Nano DNA LT Sample Prep Kit (FC-121-4001) (Illumina, San Diego, CA, USA) following the manufacturer's instructions. DNA concentration was determined by a RS232G ultraviolet-visible spectrophotometer (Eppendorf, Framingham, MA, USA) and the DNA was subsequently diluted to $1 \mathrm{ng} / \mu \mathrm{L}$ using sterile water. The universal primer sets $8 \mathrm{~F}\left(5^{\prime}\right.$-barcode+GCACCTAAYTGGGYDTAAA GNG-3') and 533R (5'-TACNVGGGTATCTAATCC-3') were used to amplify the V1-V3 hypervariable regions. All PCR reactions were carried out with Q5 DNA High-Fidelity PCR Master Mix (New England Biolabs, Ipswich, MA, USA). PCR products were quantified on a microplate reader (BioTek, FL $\times 800$, Winooski, VT, USA) using the Quant-iT PicoGreen dsDNA Assay Kit (Thermo Scientific, Waltham, MA, USA).

PCR products were subjected to electrophoresis on $2 \%$ agarose gel for detection. Samples with bright main strips between 200 and 450 bp were chosen for further experiments, and PCR products were mixed in equidensity ratios. Then, the PCR products were purified using the Axygen Gel Extraction Kit (Axygen, Union City, CA, USA). Pyrosequencing was performed on an Illumina MiSeq Reagent Kit V3 (600 cycles) platform and $2 \times 300 \mathrm{bp}$ paired-end reads were generated with double terminal sequencing.

\subsection{Operational Taxonomic Unit (OTU)-Based Sequence Analysis}

Based on the unique barcode, the sequence reads were assigned to each sample and the low quality sequence were removed. Paired-end reads were merged using FLASH (V1.2.7) [23]. After quality filtering and chimera removal, the high-quality clean tags were used to perform OTU cluster and species annotation by UPARSE software (UPARSE v7.0.1001). Sequences with $\geq 97 \%$ similarity were assigned to the same OTU. Species anno- 
tation was carried out using the UNITE database (http:/ / www.arb-silva.de/ (accessed on 1 January 2021)) [22], based on Blast algorithm, calculated by QIIME software (Version 1.7.0).

\subsection{Statistical Analyses}

The alpha diversity, performed based on OTU normalized data, was applied to analyze the complexity of species diversity in a sample using five indices, namely, observed species, diversity indices (Shannon's, Simpson's) and community richness indices (chao1, ACE). Beta diversity analysis was used to evaluate differences in species complexity between samples. Correlations between the OTU data and soil chemical properties were determined by redundancy analysis (RDA), which was carried out via the vegan package of R.

One-way analysis of variance followed by Tukey's test $(p<0.05)$ was used for multiple comparisons using SPSS 20.0. To assess the relative contribution (\%) of each microbial taxon to the dissimilarity across samples, similarity percentage analysis (SIMPER) was performed with the PRIMER V5 software package (PRIMER-E Ltd., Plymouth, UK) [23].

\section{Results}

\subsection{Yield and Lobetyolin Content}

Quantitative and qualitative differences were observed between main cropping (FLZ) and continuous cropping (LZ) samples (Table 1). The yield was significantly greater (by $11 \%$ ) in main cropping compared to that in continuous cropping. The lobetyolin content, from continuous cropping of $C$. tangshen roots, was significantly improved by $110 \%$ compared to that from main cropping.

Table 1. Comparison of yield and Lobetyolin content between main cropping (FLZ) and continuous cropping (LZ).

\begin{tabular}{ccc}
\hline Treatment & $\begin{array}{c}\text { Yield } \\
\text { (g/plant, FW) }\end{array}$ & Lobetyolin Content (mg/g) \\
\hline LZ & $667.67 \pm 15.20$ & $2.74 \pm 0.13$ \\
FLZ & $740.93 \pm 21.40^{*}$ & $1.30 \pm 0.08^{*}$ \\
\hline
\end{tabular}

Values are means \pm SDs $(n=3)$. Values followed by star symbol are significantly different between main cropping (FLZ) and continuous cropping (LZ) treatments.

\subsection{Soil Chemical Properties}

Compared to that in main cropping (FLZ) soil, organic matter content, total nitrogen, available nitrogen, total phosphorus, available phosphorus, and available potassium were higher in continuous cropping (LZ) soil that was collected on 4 May, 14 July, and 19 September 2016, consecutively (Table 2). Moreover, total potassium was significantly higher in the continuous cropping soil than in main cropping soil on 4 May 2016. However, no significant change was observed in $\mathrm{pH}$ and total potassium content between main cropping and continuous cropping soil samples on 14 July and 19 September 2016.

\subsection{Bacterial Community Composition}

16S rRNA deep pyrosequencing was performed to assess the effects of continuous cropping of $C$. tangshen on soil bacterial community. The sparse curve can judge whether the current sequencing depth of each sample is sufficient to reflect the microbial diversity contained in the community sample. Rarefaction analyses showed that the number of observed species plateau at 2395 sequences (Figure 1). Sequences from six soil samples were assigned to $1,088,000$ OTUs at the $97 \%$ similarity cut-off level. There were 660,849 and 2344 OTUs in the continuous cropping soil and main cropping soil, respectively. 
Table 2. Chemical properties of soils from different treatment plots.

\begin{tabular}{ccccccccc}
\hline & $\mathbf{p H}$ & $\begin{array}{c}\text { OMC } \\
\mathbf{g} / \mathbf{k g}\end{array}$ & $\begin{array}{c}\text { TN } \\
\mathbf{\%}\end{array}$ & $\begin{array}{c}\text { AN } \\
\mathbf{m g} / \mathbf{k g}\end{array}$ & $\begin{array}{c}\text { TP } \\
\mathbf{\%}\end{array}$ & $\begin{array}{c}\text { AP } \\
\mathbf{m g} / \mathbf{k g}\end{array}$ & $\begin{array}{c}\text { TK } \\
\mathbf{\%}\end{array}$ & $\begin{array}{c}\text { AK } \\
\mathbf{m g} / \mathbf{k g}\end{array}$ \\
\hline LZ1 & $5.64 \mathrm{~b}$ & $21.24 \mathrm{a}$ & $0.21 \mathrm{a}$ & $196.07 \mathrm{a}$ & $0.07 \mathrm{a}$ & $51.65 \mathrm{a}$ & $1.91 \mathrm{a}$ & $268.80 \mathrm{a}$ \\
FLZ1 & $6.24 \mathrm{a}$ & $15.85 \mathrm{bc}$ & $0.17 \mathrm{bc}$ & $148.35 \mathrm{~b}$ & $0.05 \mathrm{c}$ & $8.22 \mathrm{c}$ & $1.09 \mathrm{~b}$ & $104.26 \mathrm{c}$ \\
LZ2 & $5.41 \mathrm{~b}$ & $20.06 \mathrm{a}$ & $0.18 \mathrm{~b}$ & $111.70 \mathrm{c}$ & $0.06 \mathrm{ab}$ & $35.43 \mathrm{~b}$ & $1.85 \mathrm{a}$ & $239.74 \mathrm{abc}$ \\
FLZ2 & $5.37 \mathrm{~b}$ & $14.30 \mathrm{c}$ & $0.16 \mathrm{c}$ & $101.55 \mathrm{c}$ & $0.05 \mathrm{c}$ & $6.70 \mathrm{c}$ & $1.87 \mathrm{a}$ & $148.93 \mathrm{abc}$ \\
LZ3 & $5.36 \mathrm{~b}$ & $18.45 \mathrm{ab}$ & $0.18 \mathrm{~b}$ & $133.34 \mathrm{bc}$ & $0.06 \mathrm{~b}$ & $24.74 \mathrm{~b}$ & $1.88 \mathrm{a}$ & $258.60 \mathrm{ab}$ \\
FLZ3 & $5.38 \mathrm{~b}$ & $14.68 \mathrm{bc}$ & $0.16 \mathrm{c}$ & $125.39 \mathrm{bc}$ & $0.05 \mathrm{c}$ & $5.83 \mathrm{c}$ & $1.87 \mathrm{a}$ & $111.65 \mathrm{bc}$ \\
\hline
\end{tabular}

OMC, TN, AN, TP, AP, TK, AK represent organic matter contents, total nitrogen, available nitrogen, total phosphorus, available phosphorus, total potassium and available potassium, respectively. LZ1, FLZ, LZ2, FLZ2, LZ3 and FLZ3 represent the soil samples collected from continuous cropping (LZ) and main cropping (FLZ) at the 4 May (LZ1 and FLZ1), 14 July (LZ2 and FLZ2) and 19 September (LZ3 and FLZ3), 2016. Different letters in columns show significant differences determined by Tukey's test $(p<0.05, n=3)$.

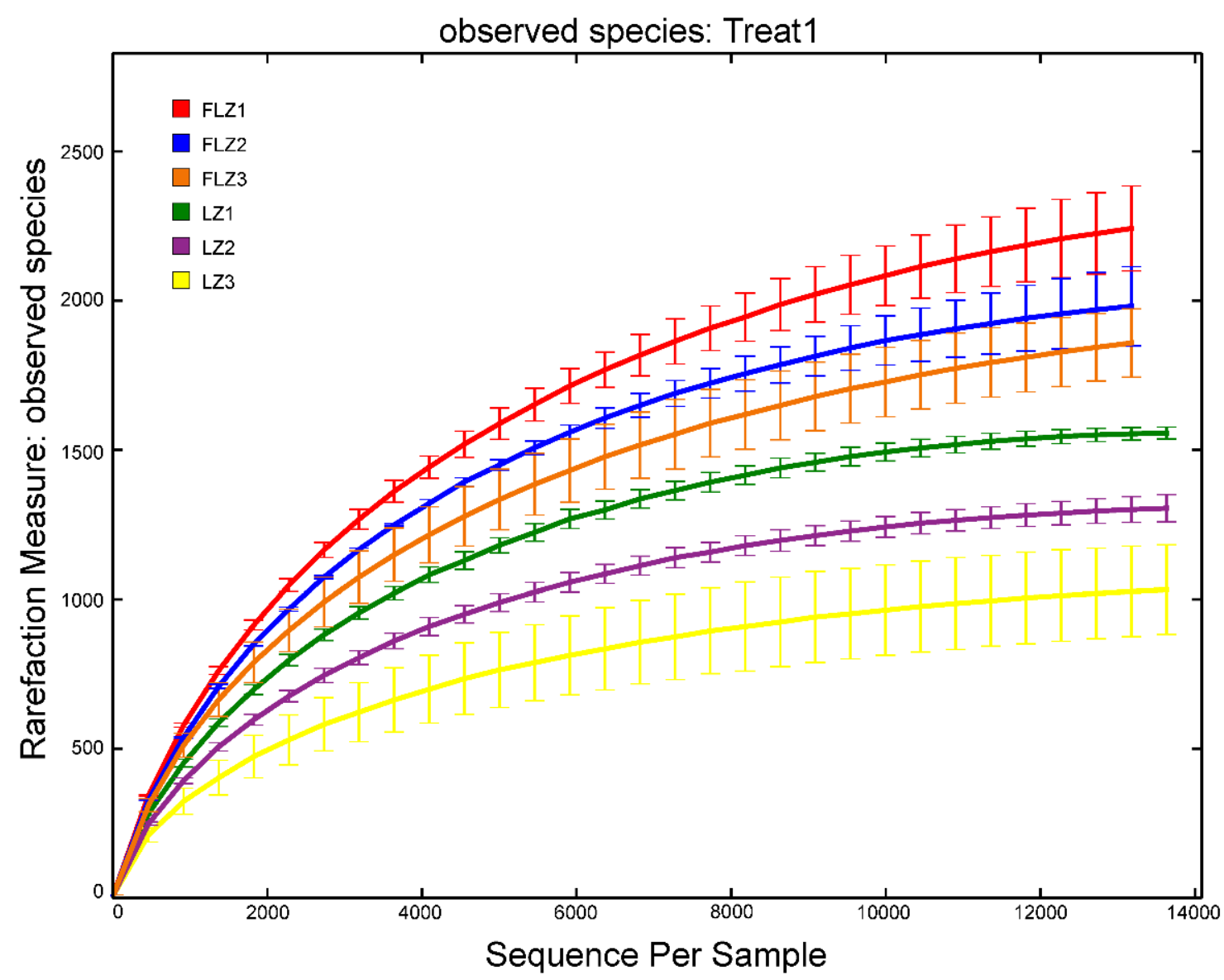

Figure 1. Rarefaction curves of bacterial communities based on observed OTUs at $97 \%$ sequence similarity for individual samples. LZ1, FLZ, LZ2, FLZ2, LZ3 and FLZ3 represent the soil samples collected from continuous cropping (LZ) and main cropping (FLZ) at the 4 May (LZ1 and FLZ1), 14 July (LZ2 and FLZ2) and 19 September (LZ3 and FLZ3), 2016.

OTU classification and their status identification results were statistically analyzed to visually compare the differences of OTU numbers and classification status in six different samples (Table 3). For bacterial communities, phylum, class, order, family, and genus were counted to reflect their community structure in different soil samples. Compared to that in the continuous cropping soil, the count of phylum, class, order, and family were significantly higher in the main cropping soil, regardless of the sampling time. However, no notable difference in genus was observed between the main cropping and continuous cropping soil on 4 May 2016. 
Table 3. Statistical table of classification and classification of status identification results by OTU in different soil samples.

\begin{tabular}{cccccc}
\hline Treatment & Phylum & Class & Order & Family & Genus \\
\hline LZ1 & $1579.66 \mathrm{c}$ & $1553.00 \mathrm{c}$ & $1357.66 \mathrm{~d}$ & $919.00 \mathrm{~b}$ & $295.00 \mathrm{ab}$ \\
FLZ1 & $2344.33 \mathrm{a}$ & $2318.33 \mathrm{a}$ & $2000.00 \mathrm{a}$ & $1176.66 \mathrm{a}$ & $341.66 \mathrm{a}$ \\
LZ2 & $1376.33 \mathrm{c}$ & $1350.66 \mathrm{c}$ & $1128.33 \mathrm{ab}$ & $763.66 \mathrm{c}$ & $255.33 \mathrm{bc}$ \\
FLZ2 & $2061.33 \mathrm{~b}$ & $2036.66 \mathrm{~b}$ & $1746.00 \mathrm{c}$ & $1061.33 \mathrm{a}$ & $348.00 \mathrm{a}$ \\
LZ3 & $1101.33 \mathrm{~d}$ & $1079.33 \mathrm{~d}$ & $903.00 \mathrm{e}$ & $588.33 \mathrm{~d}$ & $171.33 \mathrm{~d}$ \\
FLZ3 & $1956.00 \mathrm{~b}$ & $1926.00 \mathrm{~b}$ & $1600.33 \mathrm{~b}$ & $927.66 \mathrm{~b}$ & $229.66 \mathrm{c}$ \\
\hline
\end{tabular}

LZ1, FLZ, LZ2, FLZ2, LZ3 and FLZ3 represent the soil samples collected from continuous cropping (LZ) and main cropping (FLZ) at the 4 May (LZ1 and FLZ1), 14 July (LZ2 and FLZ2) and 19 September (LZ3 and FLZ3), 2016. Different letters in columns show significant differences determined by Tukey's test $(p \leq 0.05, n=3)$.

More unique OTUs were detected in the main cropping site than in continuous cropping site regardless of the sampling time. The number of OTUs exclusively found in the main cropping site was 2743 while it was only 1403 in the continuous cropping site (Figure 2).
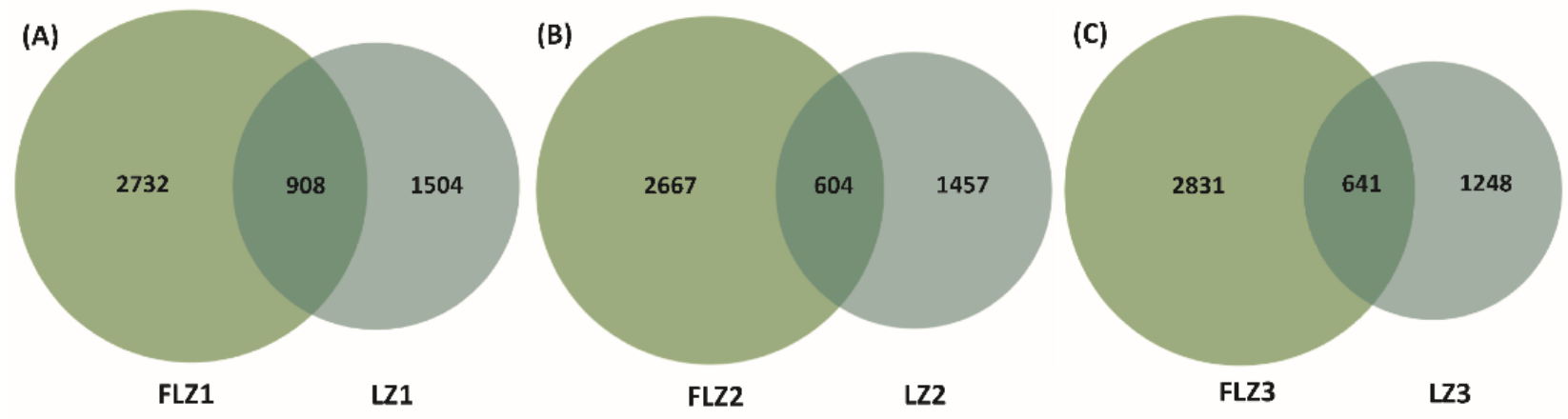

Figure 2. Venn diagram of exclusive and shared species-level taxa among the continuous cropping (LZ) and main cropping (FLZ) soils. (A) FLZ1 VS LZ1. (B) FLZ2 VS LZ2. (C) FLZ3 VS LZ3. LZ1, FLZ1, LZ2, FLZ2, LZ3 and FLZ3 represent the soil samples collected from continuous cropping (LZ) and main cropping (FLZ) at the 4 May (LZ1 and FLZ1), 14 July (LZ2 and FLZ2) and 19 September (LZ3 and FLZ3), 2016.

\subsection{Alpha Diversity Indices}

The alpha diversity indices (Chao1, ACE, Shannon index, and Simpson index) at a $3 \%$ cut-off are summarized in Table 4 . The richness of bacteria (Chao1 and ACE) between main cropping (FLZ) and continuous cropping (LZ) soil had no significant difference between 4 May and 14 July 2016. On 19 September, Chao1 and ACE indices were found to be significantly higher in main cropping soil than in continuous cropping soil $(p<0.05)$. However, the diversity of bacteria (Shannon and Simpson) between main cropping and continuous cropping soil did show a statistically significant difference. Taken together, the bacterial diversity on main cropping soil was found to be higher than that on continuous cropping soil. 
Table 4. Calculations of observed species, richness and diversity in different soil samples.

\begin{tabular}{cccccc}
\hline Treatment & $\begin{array}{c}\text { Observed } \\
\text { Species }\end{array}$ & Chao1 & ACE & Simpson & Shannon \\
\hline LZ1 & $1833.66 \mathrm{ab}$ & $2033.79 \mathrm{a}$ & $2051.47 \mathrm{ab}$ & $0.9958 \mathrm{a}$ & $9.42 \mathrm{a}$ \\
FLZ1 & $2084.66 \mathrm{a}$ & $2220.55 \mathrm{a}$ & $2303.82 \mathrm{a}$ & $0.9966 \mathrm{a}$ & $9.74 \mathrm{a}$ \\
LZ2 & $1377.00 \mathrm{bc}$ & $1459.92 \mathrm{ab}$ & $1472.48 \mathrm{ab}$ & $0.9906 \mathrm{~b}$ & $8.55 \mathrm{~b}$ \\
FLZ2 & $2061.66 \mathrm{a}$ & $2232.22 \mathrm{a}$ & $2319.05 \mathrm{a}$ & $0.9974 \mathrm{a}$ & $9.82 \mathrm{a}$ \\
LZ3 & $1097.66 \mathrm{c}$ & $1185.78 \mathrm{~b}$ & $1233.78 \mathrm{~b}$ & $0.9880 \mathrm{~b}$ & $7.99 \mathrm{~b}$ \\
FLZ3 & $1952.33 \mathrm{a}$ & $2164.32 \mathrm{a}$ & $2278.98 \mathrm{a}$ & $0.9963 \mathrm{a}$ & $9.56 \mathrm{a}$ \\
\hline
\end{tabular}

LZ1, FLZ, LZ2, FLZ2, LZ3 and FLZ3 represent the soil samples collected from continuous cropping (LZ) and main cropping (FLZ) at the 4 May (LZ1 and FLZ1), 14 July (LZ2 and FLZ2) and 19 September (LZ3 and FLZ3), 2016 Different letters in columns show significant differences determined by Tukey's test $(p \leq 0.05, n=3)$.

\subsection{Bacterial Community Structure}

The results of principal coordinate analysis (PCoA) and hierarchical cluster analysis (HCA) showed that there were significant differences in bacterial community structure between different treatments, and similar patterns for similar treatments (Figure 3). PCoA analysis revealed a separation between the soil samples from continuous cropping and main cropping by the first dimension, which represented about $68.94 \%$ of the total variance (Figure 3A). Simultaneously, HCA was applied, and it was convenient to find that all samples grouped into two major clusters corresponding to the two different regimes (continuous cropping and main cropping) (Figure 3B). Three subgroups corresponding to sampling time could be distinctly identified in the two major clusters.

(A)

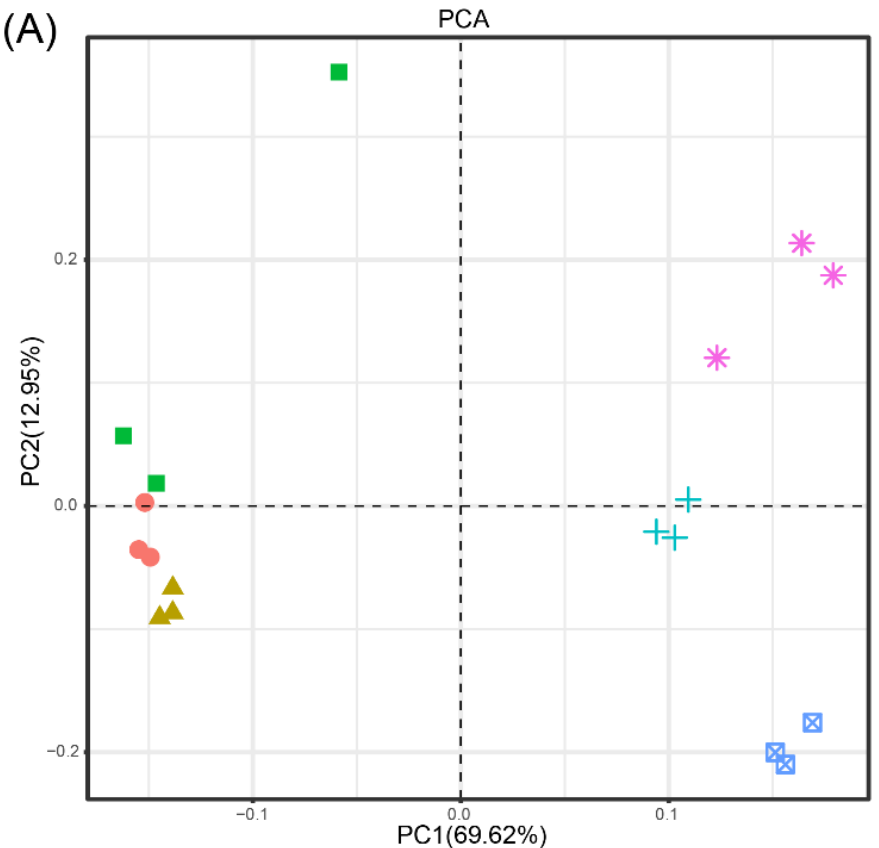

(B)

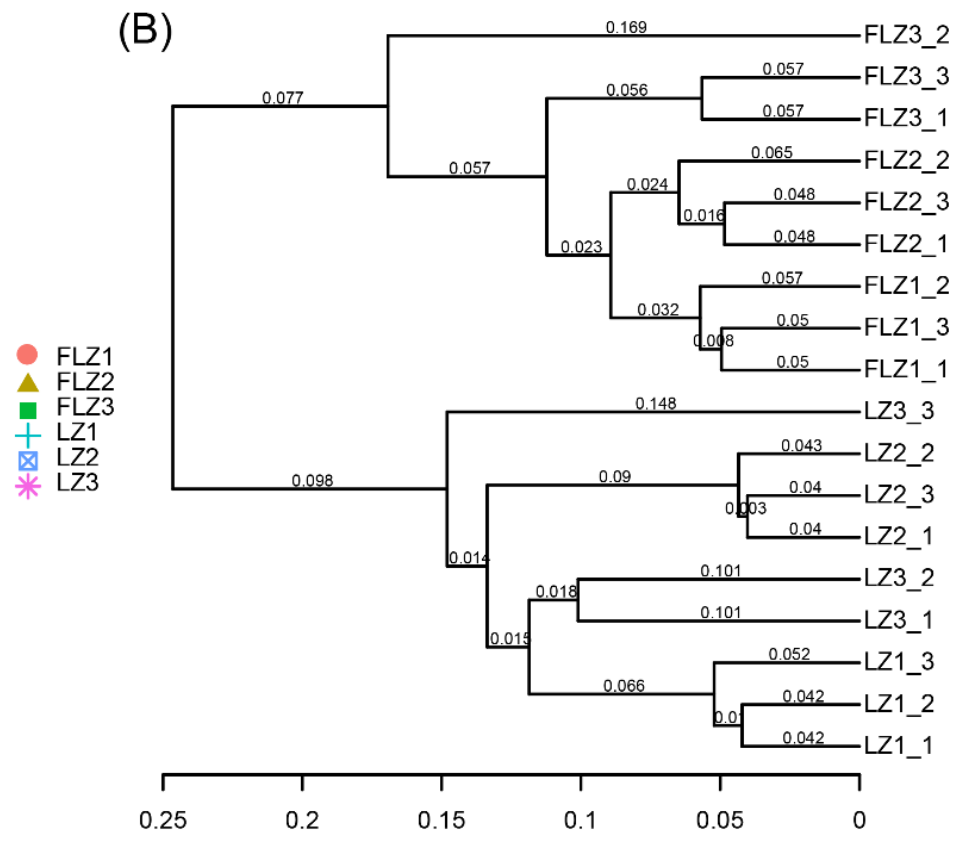

Figure 3. Principal coordinate analysis (PCoA) (A) and hierarchical clustering (B) of bacterial communities based on the weighted unifrac algorithm for six different soil sites. LZ1, FLZ, LZ2, FLZ2, LZ3 and FLZ3 represent the soil samples collected from continuous cropping (LZ) and main cropping (FLZ) at the 4 May (LZ1 and FLZ1), 14 July (LZ2 and FLZ2) and 19 September (LZ3 and FLZ3), 2016. 
A heat map analysis of the top 50 most abundant genera in six different treatments was performed at the genus level, (Figure 4). The color gradient reflects the high and low abundances of bacterial community structure across the samples. It showed that main cropping soil formed different communities than continuous cropping soil, regardless of the sampling time.

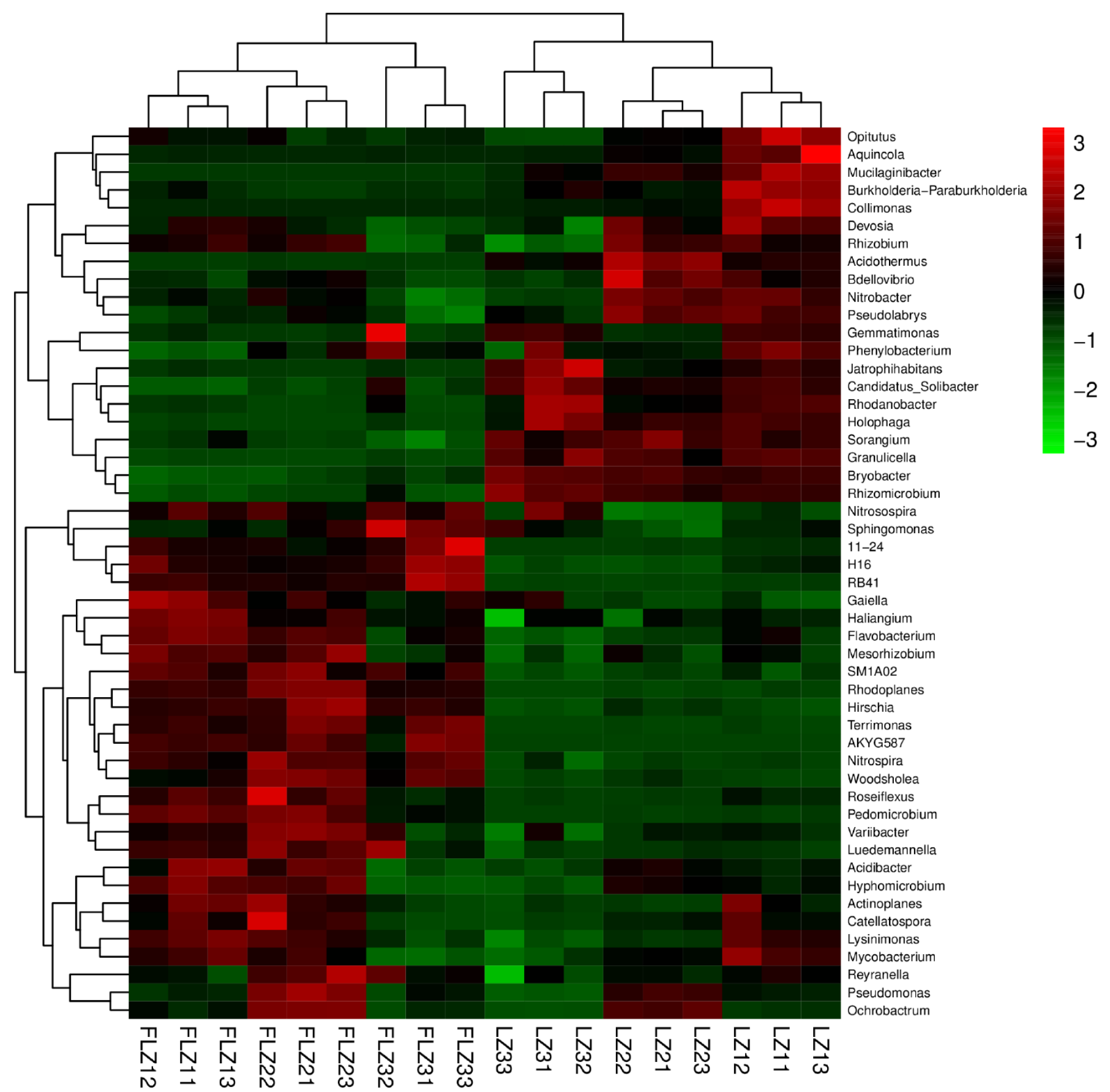

Figure 4. Heat map analysis of the top 50 most abundant genera in six different treatments. LZ1, FLZ, LZ2, FLZ2, LZ3 and FLZ3 represent the soil samples collected from continuous cropping (LZ) and main cropping (FLZ) at the 4 May (LZ1 and FLZ1), 14 July (LZ2 and FLZ2) and 19 September (LZ3 and FLZ3), 2016. 


\subsection{Shifts in Soil Bacterial Community Composition}

The phylogenetic classification of sequences, at phylum, class, and genus levels, from the six treatments is summarized in Figure 5. The bacteria OTUs were comprised mainly of seven phyla, including Proteobacteria, Acidobacteria, Gemmatimonadetes, Chloroflexi, Actinobacteria, Bacteroidetes, and Nitrospirae. Proteobacteria was the first dominant phylum, both at the continuous cropping and main cropping sites, representing $38.54 \%$ and $40.21 \%$, respectively. Chloroflexi was a dominant phylum in the main cropping site, representing $10.37 \%$, whereas it was only $5.96 \%$ in the continuous cropping site. The percentage of Acidobacteria was only $17.20 \%$ in the main cropping site, which reached $23.48 \%$ in the continuous cropping site (Figure 5A, Supplementary Table S1).

The dominant classes in both the sites include Alphaproteobacteria, Betaproteobacteria, Gammaproteobacteria, Deltaproteobacteria, Gemm-1, Gemmatimonadetes, and Actinobacteria, whereas their distribution was different significantly (Figure 5B). Alphaproteobacteria was the first dominant class both at the continuous cropping and main cropping sites, representing $22.32 \%$ and $18.25 \%$, respectively (Supplementary Table S2). At the main cropping site, Betaproteobacteria and Acidobacteria- 6 were the second and the third dominant classes with percentages of $9.10 \%$ and $8.92 \%$, respectively. At the continuous cropping site, Acidobacteria was the second dominant class with a percentage of $8.65 \%$, and Gammaproteobacteria became the third dominant class with a percentage of $7.47 \%$. At the main cropping site, Acidobacteria- 6 and Anaerolineae were the main dominant classes, representing $8.92 \%$ and $5.62 \%$, respectively. In contrast, all the percentages at continuous cropping site were $1.04 \%$ and $0.88 \%$, respectively. In addition, the percentages of Acidobacteriia, Solibacteres, and DA052 were only $0.66 \%, 1.71 \%$, and $0.11 \%$, respectively in the main cropping site, whereas it reached $8.65 \%, 5.45 \%$, and $6.29 \%$, respectively, in the continuous cropping site (Figure 5B, Supplementary Table S2).

The first three dominant genera in the main cropping site were Rhodoplanes, Nitrospira, and Sphingobium with the percentages of $3.24 \%, 2.43 \%$, and $1.68 \%$, respectively. For the continuous cropping site, the first four genera included Candidatus Koribacter, Rhodanobacter, Candidatus Solibacter, and Rhodoplanes with the percentages of $3.59 \%, 3.10 \%, 2.88 \%$, and $2.23 \%$, respectively (Figure 5C, Supplementary Table S3). In addition, there are a large number of unclassified OTUs distributed in different genera. Approximately $80 \%$ of all bacterial strains have not been identified at genus level (Figure 5C). In conclusion, obvious differences were noted both in composition and distribution of the microbial community between the main cropping and continuous cropping sites.

\subsection{Effects of Soil Chemical Properties on Bacterial Community Abundance}

The result of redundancy analysis (RDA) is showed in Figure 6. RDA of the OTU data and soil chemical properties revealed remarkable variations in bacterial community structure, both in the continuous cropping and main cropping sites. The first two RDA components (RDA1 and RDA2) could explain $83 \%$ and $10 \%$ of the total variance, respectively. The first RDA component (RDA1) separated the main cropping soils (FLZ) from the continuous cropping soils (LZ). The continuous cropping soil samples were positively correlated with the higher organic matter content (OMC) and higher available nitrogen (AN) content. The main cropping soil samples were positively correlated with soil $\mathrm{pH}$ (Figure 6). 
(A)

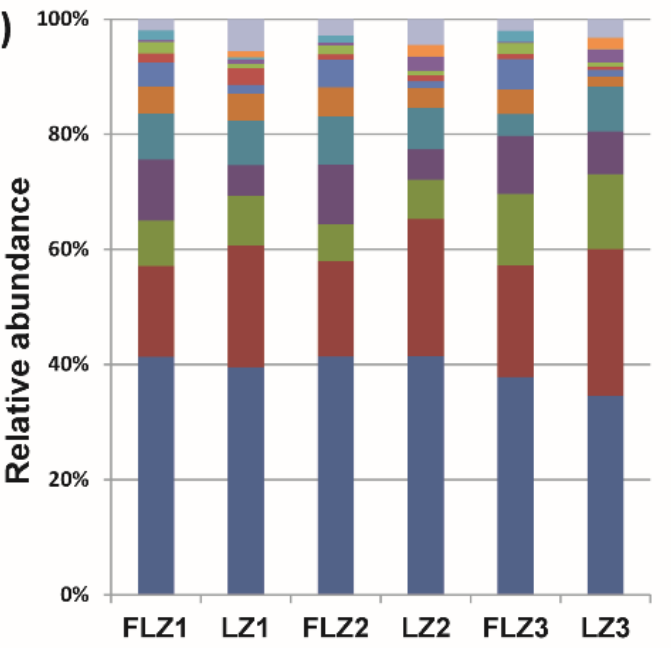

- Others

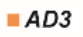

WS3

- TM7

Planctomycetes

- Verrucomicrobia

- Nitrospirae

- Bacteroidetes

Actinobacteria

- Chloroflexi

- Gemmatimonadetes

- Acidobacteria

- Proteobacteria

(B)

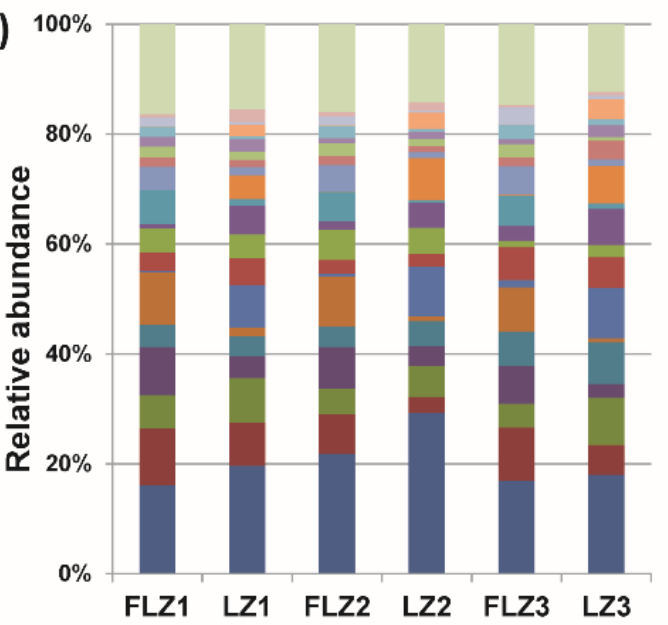

Others

Sphingobacteriia

Chloracidobacteria

Ktedonobacteria

॥ Ellin6529

- Thermoleophilia

Saprospirae

- Acidimicrobiia

= Nitrospira

- DA052

- Anaerolineae

(C)

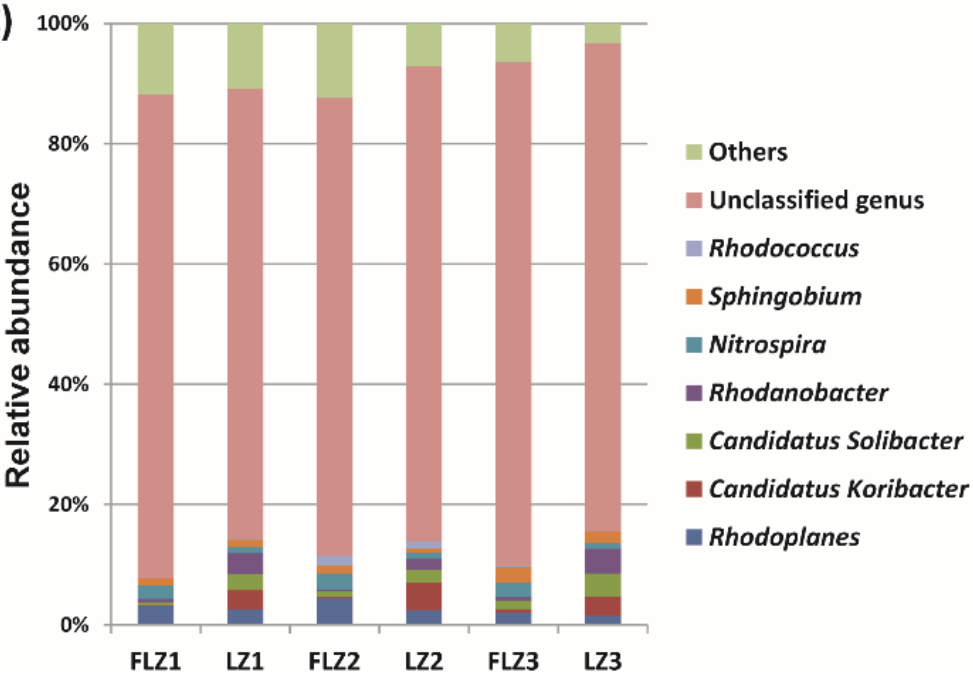

Figure 5. Bacterial community structures at (A) phylum, (B) class and (C) genus level at the six different soil sites. The relative abundance was defined as the percentage of the in total effective bacterial sequences in sample, classified using SILVA databank. Phyla, classes and genera making up less than $1 \%$ of total composition in both the libraries were classified as 'other'. LZ1, FLZ, LZ2, FLZ2, LZ3 and FLZ3 represent the soil samples collected from continuous cropping (LZ) and main cropping (FLZ) at the 4 May (LZ1 and FLZ1), 14 July (LZ2 and FLZ2) and 19 September (LZ3 and FLZ3), 2016. 


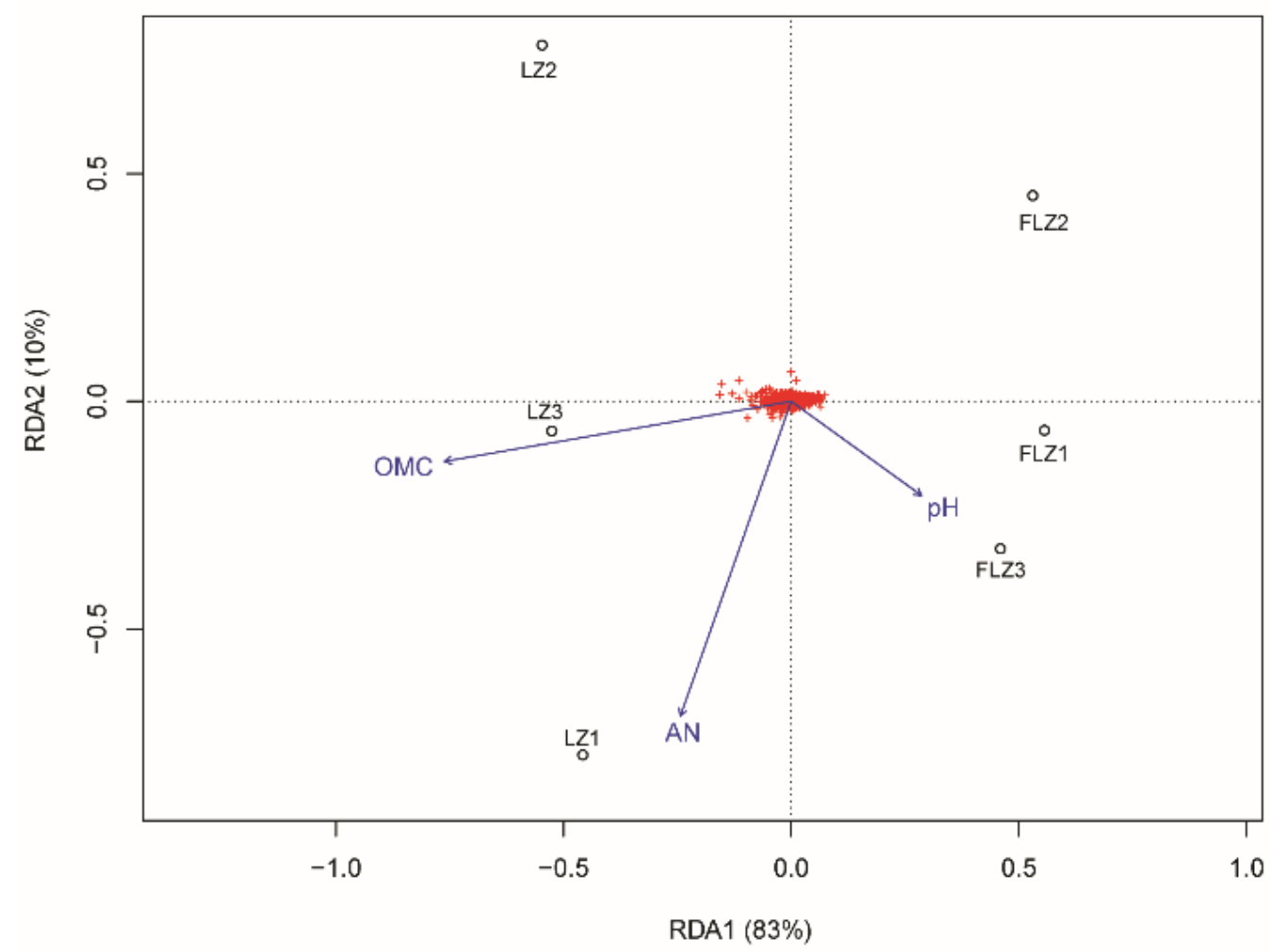

Figure 6. Redundancy analysis (RDA) of the OTUs data and soil chemical properties for individual sample from different treatments. LZ1, FLZ, LZ2, FLZ2, LZ3 and FLZ3 represent the soil samples collected from continuous cropping (LZ) and main cropping (FLZ) at the 4 May (LZ1 and FLZ1), 14 July (LZ2 and FLZ2) and 19 September (LZ3 and FLZ3), 2016. OMC and AN represent organic matter contents and available nitrogen, respectively.

\section{Discussion}

Most of the medicinal plants are obtained by cultivation; C. tangshen is a rare plant resource. One of the most common problems in crop cultivation is the obstacle of continuous cropping [3]. In recent years, with the expansion of planting area for Chinese medicinal herbs, deterioration of the environment and damage of soil due to continuous cultivation of medicinal plants has increased remarkably. Continuous cropping obstacles have become the bottleneck of many Good Agricultural Practice (GAP) based constructions, seriously affecting the sustainable production of Chinese medicinal materials. Continuous cropping obstacles are involved in 70\% of medicinal roots and rhizome herbs [24], such as ginseng [25], notoginseng [26], and Salvia miltiorrhiza [27]; C. tangshen is no exception in the process. In Banqiao C. tangshen, continuous cropping reduced the yield, reduced its quality, and aggravated its root diseases and insect pests. Our results also showed that continuous cropping led to yield reduction, with significant increase in root fresh weight (Table 1). As a primary metabolite, lobetyolin performs one of the most important pharmacological functions of $C$. tangshen; when the content of lobetyolin is higher, the quality of $C$. tangshen is better [3]. In the current study, the content of lobetyolin extracted from continuous cropping of $C$. tangshen roots was found to be significantly improved by more than twice of that from main cropping. Results demonstrated that continuous cropping is accompanied by significant plant yield reduction and lobetyolin accumulation in C. tangshen.

Recently, the role of the underground microbial community has been emphasized in overall plant performance [28,29]. Microbes are the most invisible in soil and are the most important drivers of plant health and productivity [30,31]. Bacteria, an important group of microbes in the soil ecosystem, are crucial for soil functions and plant health [32,33]. In this study, the significant difference of bacterial composition in soil from main cropping and continuous cropping of $C$. tangshen was discovered by 454 pyrosequencing analysis. 
The count of phylum, class, order, and family was significantly higher in main cropping cultivation than in continuous cropping in three time-spans (Table 3). This result suggested obvious differences both in the distribution and composition of microbial community at phylum, class, and genus levels across the main cropping and continuous cropping conditions. Moreover, there exist a variety of richness and diversity indices to reflect the alpha diversity of bacterial community. Different indices have different emphases on measuring community diversity. Richness (ACE and Chao) and diversity (Shannon and Simpson index) in the continuous cropping site were lower than those in the main cropping site (Table 4). The suppression of bacterial population in continuous cropping soil may be due to long-term continuous cropping system, toxicity, and accumulation of antimicrobial substances $[34,35]$. Soil bacterial abundance and diversity played an important role in soil function, quality, and sustainability. Hence, the loss of soil microbial abundance and diversity might have contributed to the poor growth of $C$. tangshen.

Many studies have reported that the continuous cropping obstacle problem of plants result from shifts in the soil microbial community induced by root exudates $[17,18]$. In this study, Proteobacteria (Alphaproteobacteria, Betaproteobacteria, Gammaproteobacteria, and Deltaproteobacteria) and Acidobacteria were the most abundant phyla. These results are consistent with those in another study that demonstrated Proteobacteria and Acidobacteria as the most common phyla in different agricultural systems or soil types $[33,36,37]$. Significant differences in bacterial community composition were observed between main cropping soils and continuous cropping soils. Some researchers showed $\beta$-Proteobacteria to be copiotrophic and associated with large amounts of available nutrients [37]. Acidobacteria were the most abundant in soils with very low resource availability [38]. Our results indicated that continuous cropping soils possess higher nutrient content than main cropping soils (Table 2). However, high relative abundance of Acidobacteria and low relative abundance of $\beta$-Proteobacteria were observed in continuous cropping soils. The results are inconsistent with those of previous studies [37,38]. Interestingly, a previous study had found Bacteroidetes to be a very important indicator of soil health [39]. Our results showed that the relative abundance of Bacteroidetes phyla was lower in continuous cropping soils than in main cropping soils, which agreed with several previous observations [39,40]. At the genus level, high relative abundance of Rhodanobacter and Pseudomonas ( $\gamma$-Proteobacteria) was observed in continuous cropping soils; other studies also showed Pseudomonadaceae and Rhodanobacter to be less abundant in conducive soil than in suppressive soil [41]. As an effective antagonistic endophyte for biological control, the density of Pseudomonas was closely related to plant growth [42,43]. In addition, the high relative abundance of Candidatus Koribacter and Candidatus and the low relative abundance of Nitrospira were observed in continuous cropping soils. However, the relationship between the above microbial species and continuous cropping barrier remains unclear, and their interactions in the local ecosystem would require further studies.

PCoA and HCA analyses showed that continuous cropping of $C$. tangshen strongly affected the variation in bacterial community structure. This result was consistent with previous studies, which had indicated that soil microbial community structure and composition and structure were significantly different across continuous cropping histories [44]. Therefore, we speculated that soil microbial community could be greatly affected by longterm continuous cropping of $C$. tangshen. RDA analysis of the OTU data and soil chemical properties revealed remarkable variations in bacterial community structure, both in the continuous cropping and main cropping sites, as soil variables influence the microbial community structure. Moreover, the composition and structure of the soil microbial community in the continuous cropping soils were positively correlated with the higher organic matter content $(\mathrm{OMC})$ and higher available nitrogen (AN) contents. 


\section{Conclusions}

In conclusion, continuous cropping of $C$. tangshen is accompanied by significant plant yield reduction and lobetyolin accumulation. Analysis of 16S rRNA genes of bacterial communities in continuous cropping and main cropping soils revealed that members of soil bacterial community and their structure were significantly affected by long-term continuous cropping of $C$. tangshen. All these changes might finally result in the poor growth of $C$. tangshen in continuous cropping soil. On analyzing the influence of soil properties on bacterial community structure in continuous cropping soils, we found organic matter content (OMC) and available nitrogen (AN) content to have the strongest effect on bacterial community structure of the analyzed soil.

Supplementary Materials: The following are available online at https:/ /www.mdpi.com/article/10 .3390/d13070317/s1, Figure S1: Sampling plan design drawing. Table S1: The relative abundances (\%) of the bacteria at the Phylum level. Table S2: The relative abundances (\%) of the bacteria at the class level. Table S3: The relative abundances (\%) of the bacteria at the genus level.

Author Contributions: Conceptualization, L.C., M.Z. and Y.H.; methodology, Y.X. and L.A.; software, H.L.; validation, Y.H. and W.Z.; formal analysis, Y.X.; investigation, W.Z. and L.A.; data curation, H.L.; writing —original draft preparation, Y.X. and W.Z.; writing-review and editing, Y.X.; project administration, L.C., M.Z. and Y.H.; funding acquisition, M.Z. All authors have read and agreed to the published version of the manuscript.

Funding: This research was funded by Technical Innovation Program of Hubei Province, grant number 2019ZYYD064.

Institutional Review Board Statement: Not applicable.

Informed Consent Statement: Not applicable.

Data Availability Statement: Data is contained within the article and supplementary material.

Conflicts of Interest: The authors declare no conflict of interest.

\section{References}

1. Feng, B.B.; Zhang, J.H. Compatibility of Codonopsis Radix with Different Herbs. Chin. J. Exp. Tradit. Med. Formulae 2016. [CrossRef]

2. Zhao, W.O.; Pang, L.; Dong, N.; Yang, S. LC-ESI-MS/MS analysis and pharmacokinetics of heterophyllin B, a cyclic octapeptide from Pseudostellaria heterophylla in rat plasma. Biomed. Chromatogr. BMC 2015, 29, 1693-1699. [CrossRef]

3. Zhao, Q.; Wu, Y.N.; Fan, Q.; Han, Q.Q.; Paré, P.W.; Xu, R.; Wang, Y.Q.; Wang, S.M.; Zhang, J.L. Improved Growth and Metabolite Accumulation in Codonopsis pilosula (Franch.) Nannf. by Inoculation of Bacillus amyloliquefaciens GB03. J. Agric. Food Chem. 2016, 64, 8103. [CrossRef]

4. Wang, Z.T.; Ng, T.B.; Yeung, H.W.; Xu, G.J. Immunomodulatory effect of a polysaccharide-enriched preparation of Codonopsis pilosula roots. Gen. Pharmacol. Vasc. Syst. 1996, 27, 1347-1350. [CrossRef]

5. Zhao, Y.; Wu, L.; Chu, L.; Yang, Y.; Li, Z.; Azeem, S.; Zhang, Z.; Fang, C.; Lin, W. Interaction of Pseudostellaria heterophylla with Fusarium oxysporum f.sp. heterophylla mediated by its root exudates in a consecutive monoculture system. Sci. Rep. 2015, 5, 8197. [CrossRef]

6. Wu, L.; Wang, H.; Zhang, Z.; Lin, R.; Lin, W. Comparative metaproteomic analysis on consecutively Rehmannia glutinosamonocultured rhizosphere soil. PLoS ONE 2011, 6, e20611. [CrossRef] [PubMed]

7. Huang, L.F.; Song, L.X.; Xia, X.J.; Mao, W.H.; Shi, K.; Zhou, Y.H.; Yu, J.Q. Plant-Soil Feedbacks and Soil Sickness: From Mechanisms to Application in Agriculture. J. Chem. Ecol. 2013, 39, 232-242. [CrossRef] [PubMed]

8. Zhang, S.; Raza, W.; Yang, X.; Jiang, H.; Huang, Q.; Xu, Y.; Liu, X.; Wei, R.; Shen, Q. Control of Fusarium wilt disease of cucumber plants with the application of a bioorganic fertilizer. Biol. Fertil. Soils 2008, 44, 1073. [CrossRef]

9. Harleen, K.; Rajwant, K.; Surinder, K.; Baldwin, I.T. Taking ecological function seriously: Soil microbial communities can obviate allelopathic effects of released metabolites. PLOS ONE 2009, 4, e4700.

10. el Zahar Haichar, F.; Marol, C.; Berge, O.; Rangel-Castro, J.I.; Prosser, J.I.; Balesdent, J.; Heulin, T.; Achouak, W. Plant host habitat and root exudates shape soil bacterial community structure. ISME J. 2008, 2, 1221-1230. [CrossRef]

11. Trivedi, P.; He, Z.; Van Nostrand, J.D.; Albrigo, G.; Zhou, J.; Wang, N. Huanglongbing alters the structure and functional diversity of microbial communities associated with citrus rhizosphere. ISME J. 2012, 6, 363-383. [CrossRef]

12. Berendsen, R.L.; Pieterse, C.M.J.; Bakker, P.A.H.M. The rhizosphere microbiome and plant health. Trends Plant Sci. 2012, 17, 478-486. [CrossRef] [PubMed]

13. Bennett, A.J.; Bending, G.D.; Chandler, D.; Hilton, S.; Mills, P. Meeting the demand for crop production: The challenge of yield decline in crops grown in short rotations. Biol. Rev. 2012, 87, 52-71. [CrossRef] [PubMed] 
14. Bramley, R.; Ellis, N.; Nable, R.O.; Garside, A.L. Changes in soil chemical properties under long-term sugar cane monoculture and their possible role in sugar yield decline. Aust. J. Soil Res. 1996, 34, 967. [CrossRef]

15. Wu, L.; Li, Z.; Ji, L.; Khan, M.A.; Huang, W.; Zhang, Z.; Lin, W. Assessment of shifts in microbial community structure and catabolic diversity in response to Rehmannia glutinosa monoculture. Appl. Soil Ecol. 2013, 67, 1-9. [CrossRef]

16. Wu, L.; Chen, J.; Wu, H.; Wang, J.; Wu, Y.; Lin, S.; Khan, M.U.; Zhang, Z.; Lin, W. Effects of consecutive monoculture of Pseudostellaria heterophyllaon soil fungal community as determined by pyrosequencing. Sci. Rep. 2016, 6, 26601. [CrossRef] [PubMed]

17. Zhou, X.; Yu, G.; Wu, F. Soil phenolics in a continuously monocropped cucumber (Cucumis sativus L.) system and their effects on cucumber seedling growth and soil microbial communities. Eur. J. Soil Sci. 2012, 63, 332-340. [CrossRef]

18. Li, X.; Ding, C.; Ke, H.; Zhang, T.; Zhang, Y.; Ling, Z.; Yang, Y.; Liu, J.; Wang, X. Soil sickness of peanuts is attributable to modifications in soil microbes induced by peanut root exudates rather than to direct allelopathy. Soil Biol. Biochem. 2014, 78, 149-159. [CrossRef]

19. Liu, Q.; Zhou, Y.G.; Xin, Y.H. High diversity and distinctive community structure of bacteria on glaciers in China revealed by 454 pyrosequencing. Syst. Appl. Microbiol. 2015, 38, 578-585. [CrossRef]

20. Song, D.; Cheng, X.M.; Long-Yun, L.I.; Zhong, G.Y.; Wang, Z.T. Determination of lobetyolin in root of Codonopsis tangshen from various cultivation areas by high-performance liquid chromatography. Zhongguo Zhong Yao Za Zhi 2008, 33, $2133-2135$.

21. Hedlund, A.; Witter, E.; An, B.X. Assessment of N, P and K management by nutrient balances and flows on peri-urban smallholder farms in southern Vietnam. Eur. J. Agron. 2003, 20, 71-87. [CrossRef]

22. Bentonjonesjr, J. Soil testing in the united states. Commun. Soil Sci. Plant. Anal. 1973, 4, 307-322.

23. Tanja, M.; Salzberg, S.L. FLASH: Fast length adjustment of short reads to improve genome assemblies. Bioinformatics 2011, 27, 2957-2963.

24. Tan, G.Y.; Yang, Z.L.; Yuan, Z.L.; Yang, X. Research advances in continuous cropping obstacle in medicinal plants and its management. J. Northwest A F Univ. 2012, 40, 197-204.

25. Chen, C.B.; Liu, J.Y.; Wang, Y.Y.; Yan, S.; Xu, S.Q. Allelopathy of Ginseng Rhizosphere and Its Effect on Germination of Seed. J. Jilin Agric. Univ. 2006, 28, 534-541.

26. Sun, M.; Ye, L.; Zhang, Z. Progress on the Cause of Continuous Cropping Obstacle of Panax Notoginseng and its Countermeasures. J. Mt. Agric. Biol. 2015, 40, 197-204.

27. Zhang, C.L.; Sun, Q.; Qing, Y.E. Obstacle Effect of Continuous Cropping on Salvia miltiorrhiza Growth. Acta Bot. Boreali-Occident. Sin. 2005, 25, 1029-1034.

28. Wardle, D.A.; Bardgett, R.D.; Klironomos, J.N.; Heikki, S.L.; Putten, W.H.; Van Der Wall, D.H. Ecological linkages between aboveground and belowground biota. Science 2004, 304, 1629-1633. [CrossRef]

29. Haney, C.H.; Ausubel, F.M. Microbiome. Plant microbiome blueprints. Science 2015, 349, 788-789. [CrossRef] [PubMed]

30. Heijden, M.G.A.; Van Der Bardgett, R.D.; van Straalen, N.M. The unseen majority: Soil microbes as drivers of plant diversity and productivity in terrestrial ecosystems. Ecol. Lett. 2010, 11, 296-310. [CrossRef]

31. Santhanam, R.; Luu, V.T.; Weinhold, A.; Goldberg, J.; Oh, Y.; Baldwin, I.T. Native root-associated bacteria rescue a plant from a sudden-wilt disease that emerged during continuous cropping. Proc. Natl. Acad. Sci. USA 2015, 112, E5013. [CrossRef]

32. Xu, L.; Ravnskov, S.; Larsen, J.; Nilsson, R.H.; Nicolaisen, M. Soil fungal community structure along a soil health gradient in pea fields examined using deep amplicon sequencing. Soil Biol. Biochem. 2012, 46, 26-32. [CrossRef]

33. Zhou, X.; Wu, F. Dynamics of the diversity of fungal and Fusarium communities during continuous cropping of cucumber in the greenhouse. FEMS Microbiol. Ecol. 2012, 80, 469-478. [CrossRef] [PubMed]

34. Zhao, J.; Wu, X.; Wu, T.; Dai, W.; Liu, H.; Yang, R. Analysis of unculturable bacterial communities in tea orchard soils based on nested PCR-DGGE. World J. Microbiol. Biotechnol. 2012, 28, 1967-1979. [CrossRef]

35. Li, Y.; Li, Z.; Jiang, Y.; Weng, B.; Lin, W. Variations of rhizosphere bacterial communities in tea (Camellia sinensis L.) continuous cropping soil by high-throughput pyrosequencing approach. J. Appl. Microbiol. 2016, 121, 787-799. [CrossRef]

36. Heiko, N.; Andrea, T.; Antje, W.; Christiane, W.; Ladislav, H.; Nadine, H.; Ingo, S.N.; Marion, S.; Rolf, D. Pyrosequencing-based assessment of bacterial community structure along different management types in German forest and grassland soils. PLoS ONE 2011, 6, e17000.

37. Shen, Z.; Wang, D.; Ruan, Y.; Xue, C.; Zhang, J.; Li, R.; Shen, Q. Deep 16 S rRNA Pyrosequencing Reveals a Bacterial Community Associated with Banana Fusarium Wilt Disease Suppression Induced by Bio-Organic Fertilizer Application. PLoS ONE 2014, 9 , e98420. [CrossRef]

38. Noah, F.; Bradford, M.A.; Jackson, R.B. Toward an ecological classification of soil bacteria. Ecology 2007, 88, 1354-1364.

39. Xiong, W.; Zhao, Q.; Zhao, J.; Xun, W.; Li, R.; Zhang, R.; Wu, H.; Shen, Q. Different Continuous Cropping Spans Significantly Affect Microbial Community Membership and Structure in a Vanilla-Grown Soil as Revealed by Deep Pyrosequencing. Microb. Ecol. 2015, 70, 209-218. [CrossRef]

40. Xiong, W.; Li, Z.; Liu, H.; Xue, C.; Zhang, R.; Wu, H.; Li, R.; Shen, Q. The Effect of Long-Term Continuous Cropping of Black Pepper on Soil Bacterial Communities as Determined by 454 Pyrosequencing. PLoS ONE 2015, 10, e0136946. [CrossRef] [PubMed]

41. Rodrigo, M.; Marco, K.; Irene, D.B.; Ester, D.; Menno, V.D.V.; Schneider, J.H.M.; Piceno, Y.M.; Desantis, T.Z.; Andersen, G.L.; Bakker, P.A.H.M. Deciphering the rhizosphere microbiome for disease-suppressive bacteria. Science 2011, 332, 1097-1100. 
42. Rumberger, A.; Merwin, I.A.; Thies, J.E. Microbial community development in the rhizosphere of apple trees at a replant disease site. Soil Biol. Biochem. 2007, 39, 1645-1654. [CrossRef]

43. Aravind, R.; Kumar, A.; Eapen, S.J.; Ramana, K.V. Endophytic bacterial flora in root and stem tissues of black pepper (Piper nigrum L.) genotype: Isolation, identification and evaluation against Phytophthora capsici. Lett. Appl. Microbiol. 2010, $48,58-64$. [CrossRef] [PubMed]

44. Li, X.; Ding, C.; Zhang, T.; Wang, X. Fungal pathogen accumulation at the expense of plant-beneficial fungi as a consequence of consecutive peanut monoculturing. Soil Biol. Biochem. 2014, 72, 11-18. [CrossRef] 